Article

\title{
Impact of the Surface Properties of Cellulose Nanocrystals on the Crystallization Kinetics of Poly(Butylene Succinate)
}

\author{
Hatem Abushammala ${ }^{1, * \mathbb{C}}$ and Jia Mao ${ }^{2}$ \\ 1 Fraunhofer Institute for Wood Research (WKI), Bienroder Weg 54E, 38108 Braunschweig, Germany \\ 2 Department of Mechanical Engineering, Al-Ghurair University, Dubai International Academic City, \\ P.O. Box 37374 Dubai, UAE; jia.mao@agu.ac.ae \\ * Correspondence: hatem.abushammala@wki.fraunhofer.de; Tel.: +49-531-215-5409
}

Received: 17 February 2020; Accepted: 11 March 2020; Published: 13 March 2020

\begin{abstract}
The hydrophilicity of cellulose nanocrystals (CNCs) is a major challenge for their processing with hydrophobic polymers and matrices. As a result, many surface modifications have been proposed to hydrophobize CNCs. The authors showed in an earlier study that grafting alcohols of different chain lengths onto the surface of CNCs using toluene diisocyanate (TDI) as a linker can systematically hydrophobize CNCs to a water contact angle of up to $120^{\circ}$ depending on the alcohol chain length. Then, the hydrophobized CNCs were used to mechanically reinforce poly(butylene succinate) (PBS), which is a hydrophobic polymer. As a result of hydrophobization, PBS/CNCs interfacial adhesion and the composite mechanical properties significantly improved with the increasing CNC contact angle. Continuing on these results, this paper investigates the impact of CNC surface properties on the crystallization behavior of PBS using differential scanning calorimetry (DSC). The results showed that the crystallization temperature of PBS increased from $74.7^{\circ} \mathrm{C}$ to up to $86.6^{\circ} \mathrm{C}$ as a result of CNC nucleation activity, and its value was proportionally dependent on the contact angle of the CNCs. In agreement, the nucleation activity factor $(\phi)$ estimated using Dobreva and Gutzow's method decreased with the increasing CNC contact angle. Despite the nucleation action of CNCs, the rate constant of PBS crystallization as estimated using the Avrami model decreased in general as a result of a prevailing impeding effect. This decrease was minimized with increasing the contact angle of the CNCs. The impeding effect also increased the average activation energy of crystallization, which was estimated using the Kissinger method. Moreover, the Avrami exponent (n) decreased because of CNC addition, implying a heterogeneous crystallization, which was also apparent in the crystallization thermograms. Overall, the CNC addition facilitated PBS nucleation but retarded its crystallization, and both processes were significantly affected by the surface properties of the CNCs.
\end{abstract}

Keywords: cellulose; nanocrystals; modification; poly(butylene succinate); crystallization; kinetics

\section{Introduction}

Cellulose nanocrystals (CNCs) are rod-shaped nanoparticles with a thickness of 3-10 nm and a length of few hundreds of nanometers [1]. They can be extracted from pulp fibers, microcrystalline cellulose, and wood using a variety of reagents including strong acids, bases, oxidizing agents, and ionic liquids [2-4]. CNCs have shown great potential in a wide range of applications due to their advantageous mechanical properties, high surface area, biocompatibility, and biodegradability $[5,6]$. Furthermore, the possibility to modify their surfaces has led to CNCs with a wide range of surface properties and functionalities $[7,8]$. Due to their mechanical strength, CNCs have often been used to reinforce synthetic and bio-based polymers [9-11]. One main obstacle is the poor interfacial 
adhesion between CNCs and their matrices, which results in weak interfaces and reduced mechanical reinforcement $[12,13]$.

Most of the commonly used polymers such as polyolefins are hydrophobic, while CNCs are hydrophilic. Two main approaches have been used to overcome this issue. One approach relies on the use of compatibilizers or coupling agents such as maleic anhydride-grafted-polyethylene (MAPE) for nanocellulose/polyethylene composites $[14,15]$. The other approach reduces the hydrophilicity of CNCs by reacting the surface hydroxyls with a variety of hydrophobic chemicals through relatively simple reactions such as acetylation and carbanilation or through grafting bulkier chemicals and polymers [16-18]. In a previous report by the authors, the surface properties of CNCs were tailored by grafting alcohols of different chain lengths onto their whole surface (almost all available surface hydroxyls, i.e., a degree of surface modification of ca. 100\%) using 2,4-toluene diisocyanate (TDI) as a linker (Figure 1) [19]. Four alcohols were explored: ethanol, 1-butanol, 1-hexanol, and 1-octanol. As a result of this surface modification, CNCs with a tailored water contact angle of up to $120^{\circ}$ were prepared, which was a result of growing a hydrophobic shell around the CNCs. These modified CNCs were used to reinforce poly(butylene succinate) (PBS), a hydrophobic polymer. The resultant composite showed improved interfacial adhesion, which was dependent on the alcohol chain length as confirmed by microscopic and thermomechanical investigations. This approach was proved to be simple for tailoring the surface properties of CNCs by only varying the alcohol chain length. It is also expected to improve the interfacial adhesion between CNCs and other hydrophobic matrices.

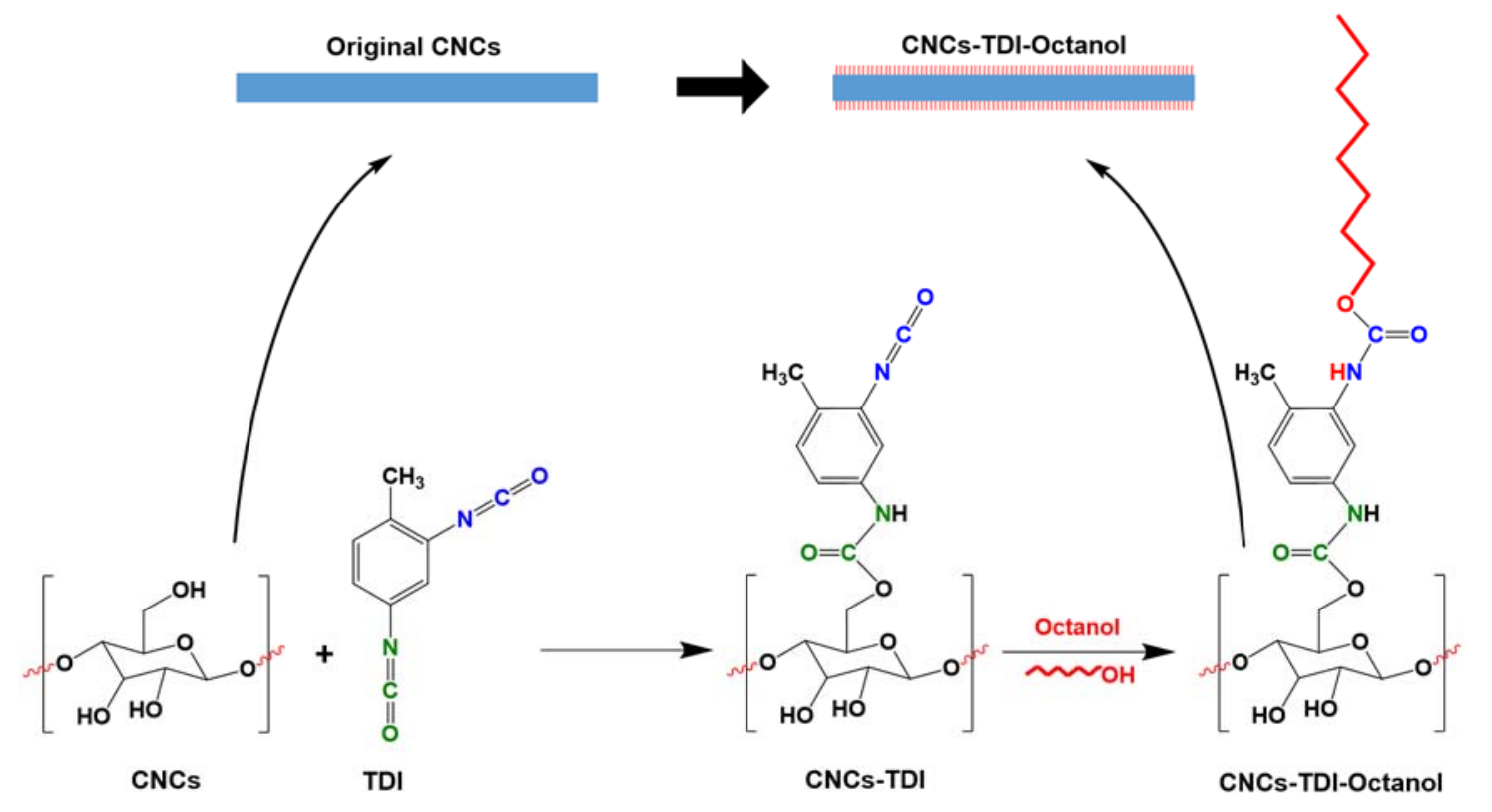

Figure 1. Tailoring the hydrophilicity of cellulose nanocrystals (CNCs) by grafting alcohols of different chain lengths on the CNC surface using toluene diisocyanate (TDI) as a linker.

PBS, which is produced by the polycondensation of succinic acid and butanediol, has shown great potential in many applications including automotive and packaging industries due to its similar properties to polyolefins and its advantageous biodegradability [20-23]. It still suffered some drawbacks in terms of its mechanical and gas barrier properties and its slow crystallization [24]. In order to modulate its properties, PBS has been processed with a variety of polymers, fibers, microparticles, and nanoparticles including CNCs, starch nanocrystals, silicon nitride, carbon nanotubes, calcium carbonate, etc. [25-27]. This resulted in significant changes in its mechanical, morphological, optical, and thermal properties [28-32], which were strongly influenced by the size and distribution of the generated spherulites upon PBS crystallization [33]. Therefore, the impact of many of these "modifiers" on the crystallization kinetics of PBS has been isothermally and non-isothermally investigated using 
Avrami and Ozawa models and their combination [34,35]. In general, the modifiers or nanofillers acted as nucleating agents, and their nucleation activity was dependent on their amount, size, and morphology [36-38]. For instance, Filizgok et al. studied the influence of the shape of different carbon-based nanoparticles on the crystallization kinetics of PBS and showed that carbon nanotubes have a stronger nucleation activity than carbon black and fullerene [39].

This paper studies the crystallization kinetics of PBS upon the addition of CNCs of different surface properties to shed more light on the impact of interfacial adhesion on the nucleation activity of CNCs in PBS composites.

\section{Materials and Methods}

\subsection{Materials}

The CNC suspension with a solid content of $10.4 \% \mathrm{w} / \mathrm{w}$ was purchased from the University of Maine, which was prepared following a sulfuric acid-mediated procedure. Ethanol, 1-butanol, 1-hexanol, 1-octanol, acetone, toluene, triethylamine (TEA), and chloroform were purchased from VWR (Darmstadt, Germany) and stored over A3 or A4 molecular sieves, which were purchased from Carl Roth (Karlsruhe, Germany) and regenerated before use. 2,4-toluene diisocyanate (2,4-TDI) was purchased from TCI Chemicals (Eschborn, Germany) and bio-based poly(butylene succinate) was purchased from PTTMCC (Bangkok, Thailand) (commercial name is BioPBS ${ }^{\mathrm{TM}}$ ).

\subsection{Hydrophobization of CNCs by Grafting Alcohols of Different Chain Lengths onto Their Surfaces}

The CNCs were reacted with TDI following the method of Habibi and Dufresne after minor modifications [40] and the optimum conditions suggested by Abushammala [41,42]. First, $9.6 \mathrm{~g}$ of $10.4 \%$ $\mathrm{CNC}$ suspension (equivalent to $1.0 \mathrm{~g}$ of dried $\mathrm{CNCs}$ ) was solvent-exchanged from water to anhydrous acetone using a washing/precipitation procedure (three times) and then to anhydrous toluene using the same procedure (twice). The precipitation was performed using a Sigma 3-16P centrifuge (5000 rpm for $30 \mathrm{~min}$ ) (Sigma Laborzentrifugen, Osterode am Harz, Germany). After the final washing with anhydrous toluene, the precipitated CNCs were transferred to a $100 \mathrm{~mL}$ round-bottom flask using $46.3 \mathrm{~mL}$ of anhydrous toluene. To them, $3.3 \mathrm{~g}$ of 2,4-TDI and $3.0 \mathrm{~mL}$ of triethylamine (TEA) as catalyst were added. The reaction proceeded at $35{ }^{\circ} \mathrm{C}$ in a nitrogen environment. After $24 \mathrm{~h}$, the reaction mixture was centrifuged to isolate the TDI-carbamated CNCs (CNCs-TDI) from the unreacted TDI and TEA. Then, the CNCs-TDI were washed three times with anhydrous toluene before transferred to 50 $\mathrm{mL}$ of anhydrous ethanol and stirred for $24 \mathrm{~h}$ at room temperature to allow a complete grafting of ethanol onto the CNC surface. Then, the CNCs were collected by centrifugation and dried at $60^{\circ} \mathrm{C}$ under vacuum to a constant mass. The reaction was repeated to assure reproducibility. The reaction was also performed using 1-butanol, 1-hexanol, and 1-octanol instead of ethanol to produced CNCs with different surface properties. The produced CNCs are referred to in this paper as CNCs-TDI-Eth, CNCs-TDI-But, CNCs-TDI-Hex, and CNCs-TDI-Oct, respectively. Following this procedure, almost every CNC surface hydroxyl had an alcohol chain attached to it. This has been confirmed in a previous paper by the authors [19].

\subsection{Water Contact Angle Measurements}

Powdered samples of the neat and modified CNCs were pressed in an FT-IR mold to obtain discs (diameter: $1 \mathrm{~cm}$ ) of smooth surfaces. The water contact angle was determined by placing a water droplet (volume of $15 \mu \mathrm{L}$ ) on each disc surface using OCA20 equipment (DataPhysics Instruments $\mathrm{GmbH}$, Filderstadt, Germany). The standard tangent procedure was used to determine the contact angle. To assure reproducibility, the measurements were performed in triplicate. 


\subsection{Processing of the Modified CNCs with PBS}

First, $0.25 \mathrm{~g}$ of the modified CNCs was dispersed in $20 \mathrm{~mL}$ of chloroform using the ultrasonicator UW2200 (power of $10 \%$ of $2200 \mathrm{~W}$ for $20 \mathrm{~s}$ ) (Bandelin Electronic, Berlin, Germany). Then, $4.75 \mathrm{~g}$ of PBS was added and stirred to a complete dissolution. The homogeneous mixture was transferred to an aluminum dish and heated gradually to $135^{\circ} \mathrm{C}$ for $24 \mathrm{~h}$ to evaporate the chloroform and melt the PBS. Then, the samples were cooled down to room temperature to obtain films containing $5 \%(w / w)$ of CNCs. They are referred to in the paper as PBS+CNCs-TDI-Eth, PBS+CNCs-TDI-But, PBS+CNCs-TDI-Hex, and PBS+CNCs-TDI-Oct. The dispersibility of the CNCs was confirmed using scanning electron microscopy and published somewhere else [19]. It is important to mention that it was not possible to prepare films using the unmodified CNCs because of their high hydrophilicity.

\subsection{Crystallization and Melting Behavior of Neat and CNC-Reinforced PBS Using Differential Scanning Calorimetry (DSC)}

The crystallization and melting behavior of the neat and CNC-reinforced PBS was investigated using the differential scanning calorimeter DSC 3+ (Mettler Toledo, Giessen, Germany). Around 10 $\mathrm{mg}$ of each sample was heated from -60 to $135^{\circ} \mathrm{C}$ in a nitrogen environment to destroy the thermal history of the sample. Afterwards, it was cooled down to $-60^{\circ} \mathrm{C}$ then heated again to $135^{\circ} \mathrm{C}$ using a heating/cooling rate of $10^{\circ} \mathrm{C} / \mathrm{min}$. This procedure was repeated using different heating/cooling rates of 8,6 , and $4^{\circ} \mathrm{C} / \mathrm{min}$, and the measurements were performed in duplicate to assure reproducibility. The crystallization temperature $\left(T_{c}\right)$ and melting temperature $\left(T_{m}\right)$ were determined as the peak of the crystallization and melting curves, respectively. The degree of crystallinity of PBS (X) was calculated from the enthalpy of melting $\left(\Delta H_{m}\right)$ of the sample in comparison to the enthalpy of melting of $100 \%$ crystalline PBS (110.3 J/g) [43]. For the CNC-reinforced PBS samples, the $\Delta H_{m}$ values were adjusted to account for CNC mass.

\section{Results and Discussion}

A previous work by the authors has shown that grafting alcohols of different chain lengths onto the surface of CNCs using TDI as a linker is a relatively simple process for tailoring CNC surface properties [19]. As a result of this grafting, the water contact angle of the modified CNCs increased to $34^{\circ} \pm 4,52^{\circ} \pm 3,104^{\circ} \pm 1$, or $120^{\circ} \pm 5$ using ethanol, 1-butanol, 1-hexanol, or 1-octanol, respectively. In comparison, the water contact angle of PBS is $77^{\circ} \pm 3$. This systematic reduction in hydrophilicity allowed the dispersion of CNCs in hydrophobic matrices such as PBS, which is in general not possible for unmodified CNCs. Moreover, thermomechanical studies showed that the surface modification had a direct positive impact on the reinforcement capabilities of the $\mathrm{CNCs}$, as it improved their interfacial adhesion with PBS. The improvement increased with increasing the chain length of the grafted alcohol. In this paper, we performed further studies to investigate the impact of interfacial adhesion on the crystallization kinetics of PBS using the modified CNCs as filler.

The non-isothermal crystallization and melting behaviors of the neat and CNC-reinforced PBS were studied using DSC at four cooling/heating rates $\left(4,6,8\right.$, and $10^{\circ} \mathrm{C} / \mathrm{min}$ ) (Figure 2 and Table 1$)$. The results showed that crystallization took place at higher temperatures for the reinforced PBS compared to the neat one due to the nucleation activity of the modified CNCs. A similar behavior has been observed for PBS composites using other nanofillers such as carbon nanotubes [39]. It is interesting that the nucleation activity of the modified CNCs was dependent on the chain length of the grafted alcohol. Using a cooling rate of $10^{\circ} \mathrm{C} / \mathrm{min}$, the crystallization temperature $\left(\mathrm{T}_{\mathrm{c}}\right)$ of the reinforced PBS was 82.2, 83.4, 84.5, and $86.6^{\circ} \mathrm{C}$ using CNCs-TDI-Eth, CNCs-TDI-But, CNCs-TDI-Hex, and CNCs-TDI-Oct compared to $74.7^{\circ} \mathrm{C}$ for neat PBS. This means a stronger interaction between PBS molecular chains and the modified CNCs with higher degrees of hydrophobization. The homogeneity of the crystallization process was also affected by the addition of the CNCs. Compared to the typical single crystallization peak of the neat PBS, the crystallization peak of the reinforced PBS had shoulders on its both sides. These shoulders indicate a special interaction between the CNCs and PBS during nucleation (first 
shoulder) and secondary crystallization (second shoulder), which resulted in accelerating both of them. However, the shape of the primary crystallization peak does not look significantly different for the neat and reinforced PBS despite taking place at significantly higher temperatures. This implies that the interaction of the CNCs with PBS changes as crystallization progresses.
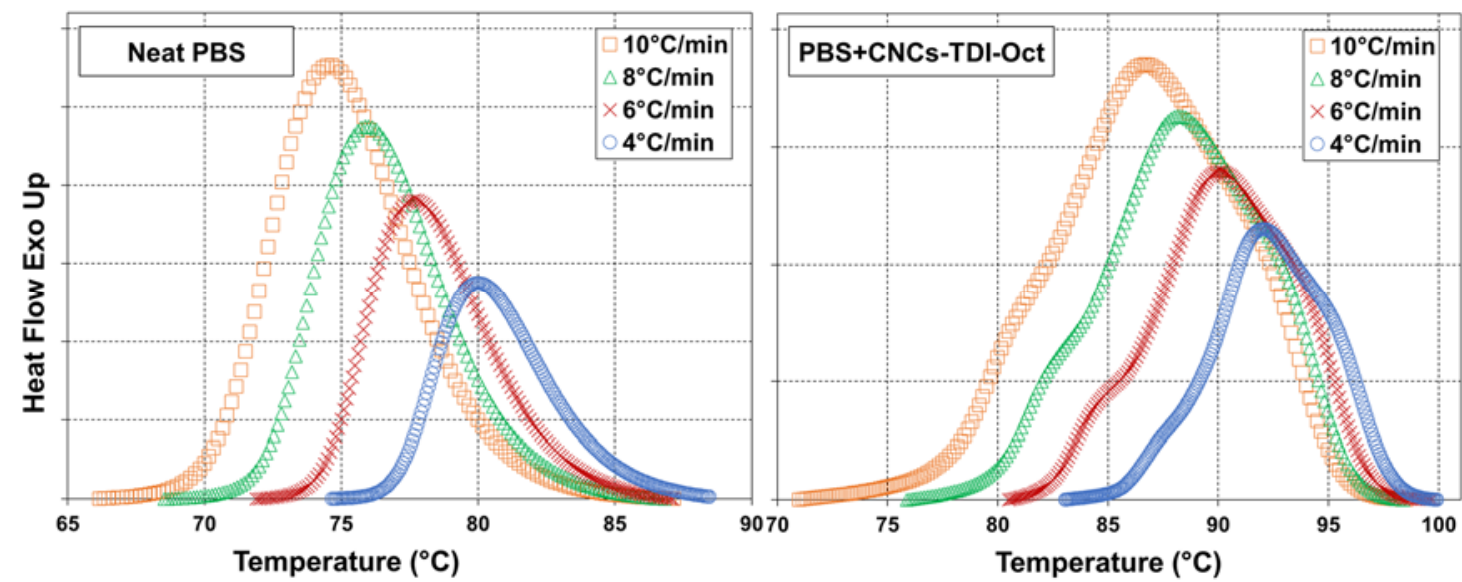

Figure 2. The crystallization exotherms of neat and reinforced poly(butylene succinate) (PBS (left)) (PBS+CNCs-TDI-Oct (right)) at different cooling rates $\left(4,6,8\right.$, and $\left.10^{\circ} \mathrm{C} / \mathrm{min}\right)$.

Table 1. The crystallization and melting parameters of neat and reinforced PBS at different cooling rates.

\begin{tabular}{|c|c|c|c|c|c|}
\hline Sample & B $\left({ }^{\circ} \mathrm{C} / \mathrm{min}\right)$ & $\mathrm{T}_{\mathrm{c}}\left({ }^{\circ} \mathrm{C}\right)$ & $\mathrm{T}_{\mathrm{m}}\left({ }^{\circ} \mathrm{C}\right)$ & $\Delta \mathrm{H}_{\mathrm{m}}(\mathrm{J} / \mathrm{g}$ PBS $)$ & $X(\%)$ \\
\hline \multirow{4}{*}{ PBS } & 4 & $80.2 \pm 0.1$ & $115.7 \pm 0.0$ & $55.1 \pm 0.4$ & $49.9 \pm 0.4$ \\
\hline & 6 & $78.0 \pm 0.3$ & $115.6 \pm 0.0$ & $56.2 \pm 0.1$ & $51.0 \pm 0.1$ \\
\hline & 8 & $76.2 \pm 0.1$ & $115.5 \pm 0.0$ & $59.6 \pm 0.2$ & $54.0 \pm 0.2$ \\
\hline & 10 & $74.7 \pm 0.1$ & $115.7 \pm 0.1$ & $62.3 \pm 0.5$ & $56.5 \pm 0.5$ \\
\hline \multirow{4}{*}{ PBS+CNCs-TDI-Eth } & 4 & $85.8 \pm 0.1$ & $115.6 \pm 0.1$ & $53.8 \pm 0.6$ & $48.8 \pm 0.5$ \\
\hline & 6 & $84.6 \pm 0.1$ & $115.6 \pm 0.0$ & $55.6 \pm 0.1$ & $50.4 \pm 0.1$ \\
\hline & 8 & $83.4 \pm 0.2$ & $115.7 \pm 0.1$ & $56.6 \pm 0.7$ & $51.3 \pm 0.6$ \\
\hline & 10 & $82.2 \pm 0.1$ & $115.8 \pm 0.0$ & $58.9 \pm 0.4$ & $53.4 \pm 0.3$ \\
\hline \multirow{4}{*}{ PBS+CNCs-TDI-But } & 4 & $87.6 \pm 0.0$ & $115.7 \pm 0.1$ & $55.6 \pm 0.9$ & $50.4 \pm 0.8$ \\
\hline & 6 & $85.8 \pm 0.0$ & $115.5 \pm 0.0$ & $57.0 \pm 0.4$ & $51.7 \pm 0.3$ \\
\hline & 8 & $84.6 \pm 0.3$ & $115.6 \pm 0.0$ & $58.1 \pm 0.5$ & $52.6 \pm 0.4$ \\
\hline & 10 & $83.4 \pm 0.1$ & $115.9 \pm 0.1$ & $58.8 \pm 1.1$ & $53.3 \pm 1.0$ \\
\hline \multirow{4}{*}{ PBS+CNCs-TDI-Hex } & 4 & $88.6 \pm 0.1$ & $115.5 \pm 0.3$ & $56.2 \pm 0.5$ & $50.9 \pm 0.4$ \\
\hline & 6 & $87.1 \pm 0.1$ & $115.6 \pm 0.3$ & $59.3 \pm 0.4$ & $53.8 \pm 0.3$ \\
\hline & 8 & $85.1 \pm 0.2$ & $115.7 \pm 0.4$ & $63.3 \pm 0.9$ & $57.4 \pm 0.9$ \\
\hline & 10 & $84.5 \pm 0.3$ & $115.8 \pm 0.5$ & $65.3 \pm 0.3$ & $59.2 \pm 0.3$ \\
\hline \multirow{4}{*}{ PBS+CNCs-TDI-Oct } & 4 & $91.7 \pm 0.4$ & $115.7 \pm 0.1$ & $56.8 \pm 0.5$ & $51.5 \pm 0.5$ \\
\hline & 6 & $89.5 \pm 0.6$ & $115.5 \pm 0.0$ & $59.7 \pm 0.8$ & $54.2 \pm 0.7$ \\
\hline & 8 & $87.9 \pm 0.4$ & $115.6 \pm 0.0$ & $64.9 \pm 1.4$ & $58.8 \pm 1.3$ \\
\hline & 10 & $86.6 \pm 0.1$ & $115.9 \pm 0.1$ & $67.3 \pm 1.0$ & $61.0 \pm 0.9$ \\
\hline
\end{tabular}

In terms of PBS melting behavior, the melting temperature was not affected by the CNCs (around $115^{\circ} \mathrm{C}$ for all samples). However, the crystallinity was slightly affected. The PBS samples reinforced by CNCs-TDI-Eth and CNCs-TDI-But showed a slightly lower crystallinity than neat PBS, while those reinforced by CNCs-TDI-Hex and CNCs-TDI-Oct showed higher crystallinity. It was dependent on the alcohol chain length of the modified CNCs, which could be a result of the higher nucleation activity of the modified CNCs with increasing the chain length of the grafted alcohol. Overall, the crystallinity values are in accordance with those reported in the literature [34]. 
To estimate the nucleation activity of nucleating agents, a simple method was proposed by Dobreva and Gutzow [44]. They proposed the nucleation activity factor $\phi$, which varies from 0 to 1 and represents the ratio between the heterogeneous and homogenous nucleation parameters, $B^{*}$ and $B$, respectively $\left(\phi=B^{*} / B\right)$. $B^{*}$ and $B$ can be estimated by plotting $\ln \beta$ versus the reciprocal of $\left(T_{m}-T_{c}\right)^{2}$ according to the following equation ( $\mathrm{C}$ is a constant):

$$
\ln \beta=-\frac{B}{\left(T_{m}-T_{c}\right)^{2}}+C .
$$

when applied on the crystallization of neat and CNC-reinforced PBS, straight lines were obtained (Figure 3), and the slope was either the B value for the neat PBS or the $\mathrm{B}^{*}$ value for the reinforced PBS. Then, the nucleation activity factor was calculated (Table 2). According to the results, it is clear that PBS nucleation was accelerated in the presence of $\mathrm{CNCs}$ in general, as the $\mathrm{B}^{*}$ values of the reinforced PBS were all lower than the $B$ value of neat PBS. Moreover, the nucleation activity was dependent on the surface properties of the CNCs as it increased with increasing the chain length of the grafted alcohol. This implies that stronger PBS/CNCs interfacial adhesion results in stronger nucleation activity, as suggested earlier in Table 1.

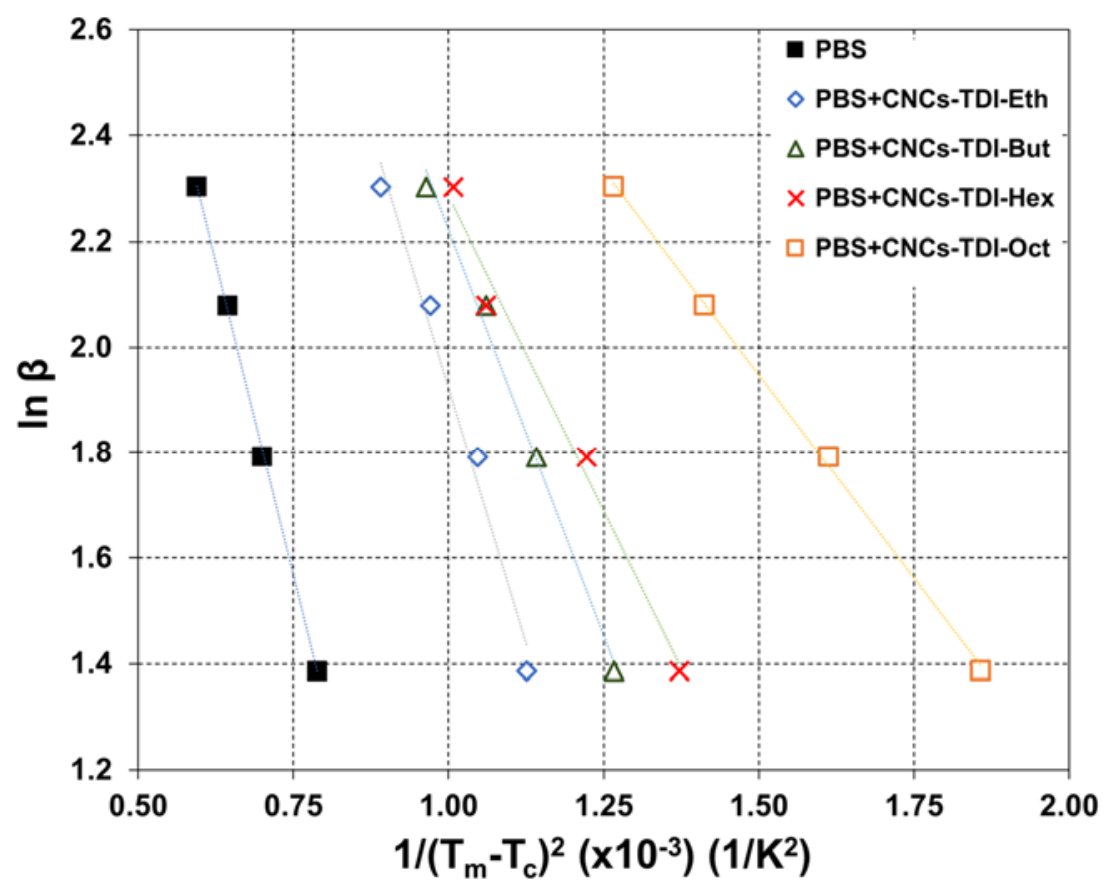

Figure 3. Nucleation activity determination for the neat and reinforced PBS using Dobreva and Gutzow's method.

Table 2. The estimated nucleation activity values for neat and reinforced PBS.

\begin{tabular}{ccc}
\hline Sample & B or B $^{*}$ & $\boldsymbol{\phi}$ \\
\hline PBS & $4638 \pm 112$ & - \\
PBS+CNCs-TDI-Eth & $3821 \pm 62$ & 0.82 \\
PBS+CNCs-TDI-But & $2907 \pm 177$ & 0.63 \\
PBS+CNCs-TDI-Hex & $2398 \pm 16$ & 0.52 \\
PBS+CNCs-TDI-Oct & $1605 \pm 63$ & 0.35 \\
\hline
\end{tabular}

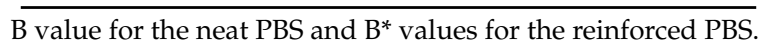


To shed more light on the interaction between the modified CNCs and PBS, the crystallization kinetics of the composite samples were studied using the Avrami model [45]. It is one of the most commonly used models to describe the crystallization process of semi-crystalline polymers. It expresses the relative crystallinity of a polymer $(X(t))$ as a function of time $(t)$ according to the following equation (where $\mathrm{Z}$ is the crystallization rate constant and $\mathrm{n}$ is the Avrami exponent) [46]:

$$
X(t)=1-\exp \left(-Z t^{n}\right)
$$

The Avrami exponent describes the mechanism of the crystallization process, and it is a term of two components: the dimensionality of crystal growth $\left(\mathrm{n}_{\mathrm{d}}\right)$ and the time dependence of nucleation $\left(n_{n}\right)$. The value of $n_{d}$ can be 1, 2, or 3 depending on if the crystal growth takes place in 1D, 2D, or $3 \mathrm{D}$, respectively. The value of $n_{n}$ is close to 1 when nucleation is homogenous and close to 0 when nucleation is heterogeneous. As a result, the value of $n$ is in the range from 1 to $4\left(n_{d}+n_{n}\right)$ [47]. The crystallization rate constant and Avrami exponent can be estimated from the slope and intercept of the logarithmic version of Avrami equation [48]:

$$
\ln [-\ln (1-X(t))]=n \ln t+\ln Z .
$$

The crystallization profiles of PBS and reinforced PBS at different cooling rates were all sigmoidal similar to the profiles of many other semi-crystalline polymers (Figure 4) [49]. The sigmoidal shape represents the different stages of crystallization. At first, nucleation takes place (first $60 \mathrm{~s}$ ) followed by a rapid crystal growth (next 60-120 s) and ends by secondary crystallization (the last 30-60 s) [50]. Expectedly, crystallization took a shorter time to completion using higher cooling rates. It was also observed that the heterogeneity of crystallization seen in Figure 2 for the reinforced PBS did not significantly affect the smoothness of its crystallization profile because the relative crystallinity is cumulative. During the nucleation stage (first $60 \mathrm{~s}$ ), the reinforced PBS samples reached higher relative crystallinity compared to neat PBS as a result of the nucleation action of the CNCs. This may explain the right shoulder of the crystallization peak of the reinforced PBS samples (Figure 2). However, crystallization took a longer time as a result of the hindrance imposed on the motion of the PBS chains by the surrounding CNCs (impeding effect).
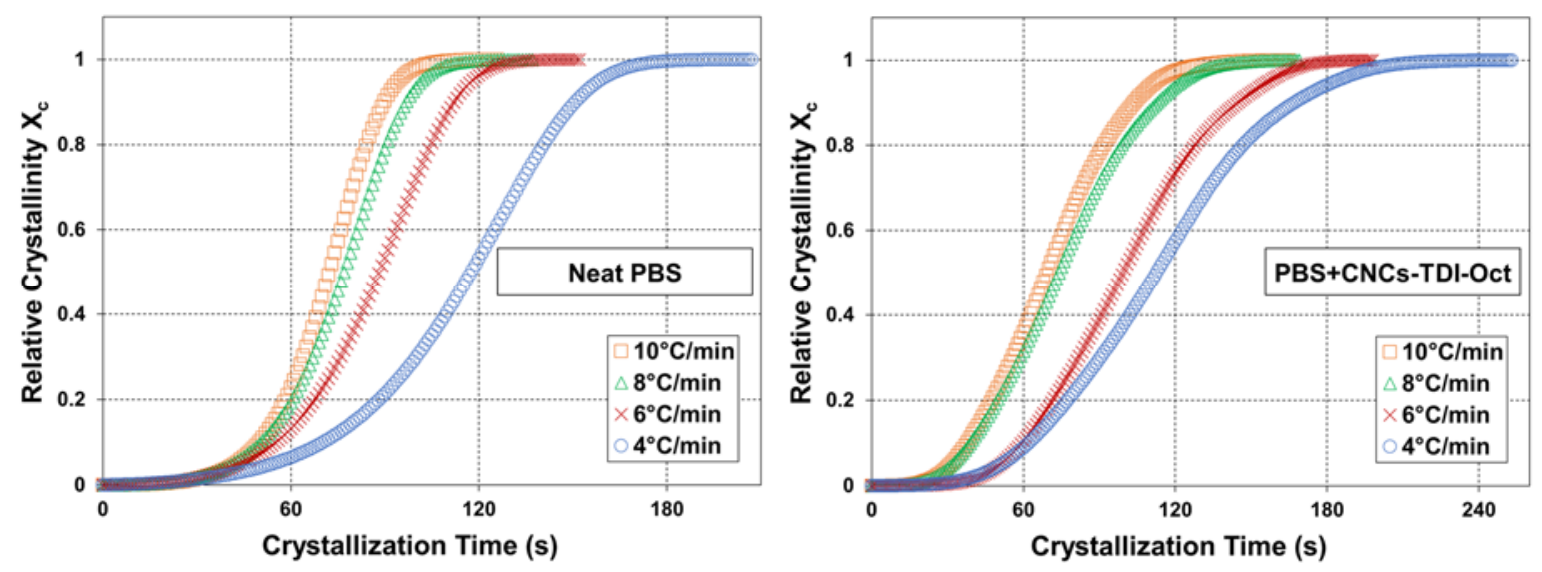

Figure 4. Plots of relative crystallinity with time at different cooling rates for neat PBS (left) and PBS+CNCs-TDI-Oct (right).

To quantitatively assess the impact of the CNCs on PBS crystallization, the Avrami kinetic parameters were estimated by plotting the logarithmic version of the Avrami equation (Figure 5 and Table 3). The crystallization of the reinforced PBS samples followed the Avrami model better than neat PBS. It deviated mainly in the beginning due to the slow nucleation of neat PBS (as also observed in Figure 2) and due to ignoring the role of secondary crystallization in the Avrami 
model [46]. In the reinforced PBS samples, the nucleation activity of the CNCs compensated for this deviation by accelerating PBS nucleation. The Avrami exponent for the neat PBS decreased upon CNC reinforcement, indicating increased crystallization heterogeneity. Avrami exponent values close to 4 imply a three-dimensional crystal growth and homogeneous nucleation (neat PBS), while values closer to 3 implies also three-dimensional growth but following a heterogeneous nucleation (reinforced PBS). This is in accordance with the crystallization curves of the neat and reinforced PBS in Figure 2. When it comes to the crystallization rate constant $(\mathrm{Z})$, it was lower for the reinforced PBS samples compared to the neat PBS due to the hindrance imposed by the CNCs on the migration and diffusion of the PBS chains to the growing crystals [51]. This was also evident from the higher half-time of crystallization $\left(t_{\frac{1}{2}}\right)$ values. This hindrance became less significant by reducing the hydrophilicity of the CNCs. It was the highest for the PBS sample reinforced by CNCs-TDI-Eth and diminished for the sample reinforced by CNCs-TDI-Oct. This may imply an optimum interfacial adhesion between PBS and CNCs-TDI-Oct, which supported a hindrance-free mobility of PBS chains during crystallization.
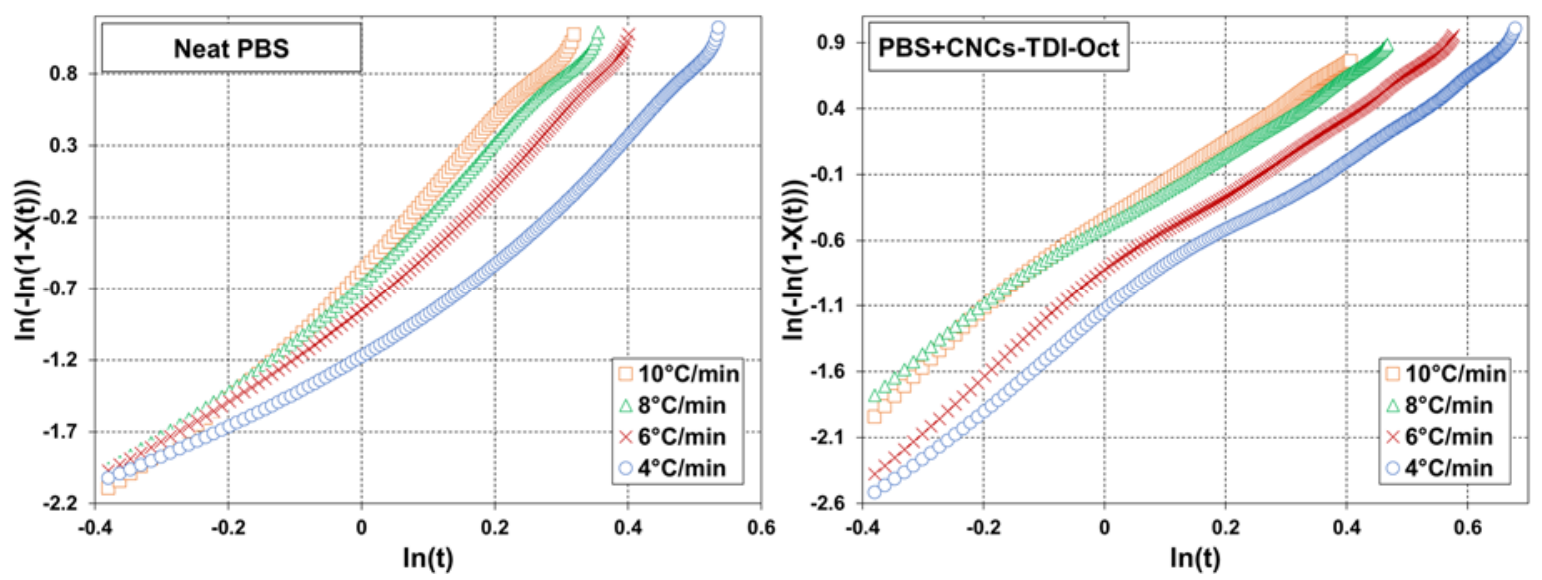

Figure 5. Avrami curves describing the crystallization of neat PBS (left) and PBS+CNCs-TDI-Oct (right) at different cooling rates.

Table 3. Avrami crystallization kinetics parameters of neat and reinforced PBS at different cooling rates.

\begin{tabular}{ccccc}
\hline Sample & $\mathbf{B}\left({ }^{\circ} \mathbf{C} / \mathbf{m i n}\right)$ & $\mathbf{n}$ & $\mathbf{Z}$ & $\mathbf{t}_{\frac{1}{2}}$ (min) \\
\hline \multirow{2}{*}{ PBS } & 4 & $3.6 \pm 0.1$ & $0.05 \pm 0.03$ & $2.0 \pm 0.1$ \\
& 6 & $3.7 \pm 0.0$ & $0.19 \pm 0.03$ & $1.5 \pm 0.1$ \\
& 8 & $3.8 \pm 0.1$ & $0.30 \pm 0.02$ & $1.3 \pm 0.1$ \\
PBS+CNCs-TDI-Eth & 10 & $3.8 \pm 0.0$ & $0.39 \pm 0.02$ & $1.1 \pm 0.1$ \\
\hline & 4 & $3.4 \pm 0.0$ & $0.03 \pm 0.00$ & $3.0 \pm 0.0$ \\
& 6 & $3.4 \pm 0.0$ & $0.11 \pm 0.04$ & $2.1 \pm 0.0$ \\
PBS+CNCs-TDI-But & 10 & $3.4 \pm 0.0$ & $0.12 \pm 0.01$ & $1.7 \pm 0.1$ \\
& 4 & $3.3 \pm 0.0$ & $0.29 \pm 0.03$ & $1.4 \pm 0.0$ \\
\hline & 6 & $3.4 \pm 0.1$ & $0.03 \pm 0.01$ & $2.6 \pm 0.2$ \\
PBS+CNCs-TDI-Hex & 10 & $3.2 \pm 0.2$ & $0.08 \pm 0.01$ & $1.9 \pm 0.1$ \\
& 4 & $3.7 \pm 0.2$ & $0.20 \pm 0.02$ & $1.7 \pm 0.0$ \\
& 6 & $3.5 \pm 0.2$ & $0.32 \pm 0.03$ & $1.3 \pm 0.0$ \\
\hline & 10 & $3.7 \pm 0.1$ & $0.07 \pm 0.01$ & $2.6 \pm 0.0$ \\
& 4 & $3.4 \pm 0.1$ & $0.10 \pm 0.02$ & $1.7 \pm 0.0$ \\
& $3.1 \pm 0.2$ & $0.31 \pm 0.04$ & $1.3 \pm 0.1$ \\
& $3.0 \pm 0.1$ & $0.33 \pm 0.05$ & $1.1 \pm 0.0$ \\
\hline & 6 & $3.2 \pm 0.1$ & $0.03 \pm 0.01$ & $2.0 \pm 0.2$ \\
& 10 & $3.6 \pm 0.3$ & $0.14 \pm 0.01$ & $1.7 \pm 0.0$ \\
& & $3.1 \pm 0.0$ & $0.30 \pm 0.02$ & $1.3 \pm 0.0$ \\
& $3.4 \pm 0.1$ & $0.40 \pm 0.04$ & $1.1 \pm 0.1$ \\
\hline
\end{tabular}


It is possible to estimate the average activation energy $\left(E_{a}\right)$ of crystallization for neat and reinforced PBS following Kissinger's method ( $R$ is the universal gas constant and $\beta_{o}$ is the exponential factor):

$$
\ln \beta=-\frac{E_{a}}{R * T_{c}}+\ln \beta_{o}
$$

The estimated crystallization activation energy of neat PBS was $169 \pm 5 \mathrm{~kJ} / \mathrm{mol}$ (Figure 6). This value is in accordance with the values reported in the literature [36]. It increased to $266 \pm 16 \mathrm{~kJ} / \mathrm{mol}$ when PBS was reinforced by CNCs-TDI-Eth. This implies that crystallization was hindered, although the addition of the modified CNCs in general facilitated PBS nucleation. However, the activation energy was dependent on the surface properties of the CNCs. It decreased with increasing the chain length of the grafted alcohol. The activation energy was $236 \pm 8,225 \pm 5$, and $196 \pm 11 \mathrm{~kJ} / \mathrm{mol} \mathrm{using}$ CNCs-TDI-But, CNCs-TDI-Hex, and CNCs-TDI-Oct, respectively. These conclusions agree with those made based on the estimated Avrami kinetics parameters (Table 2).

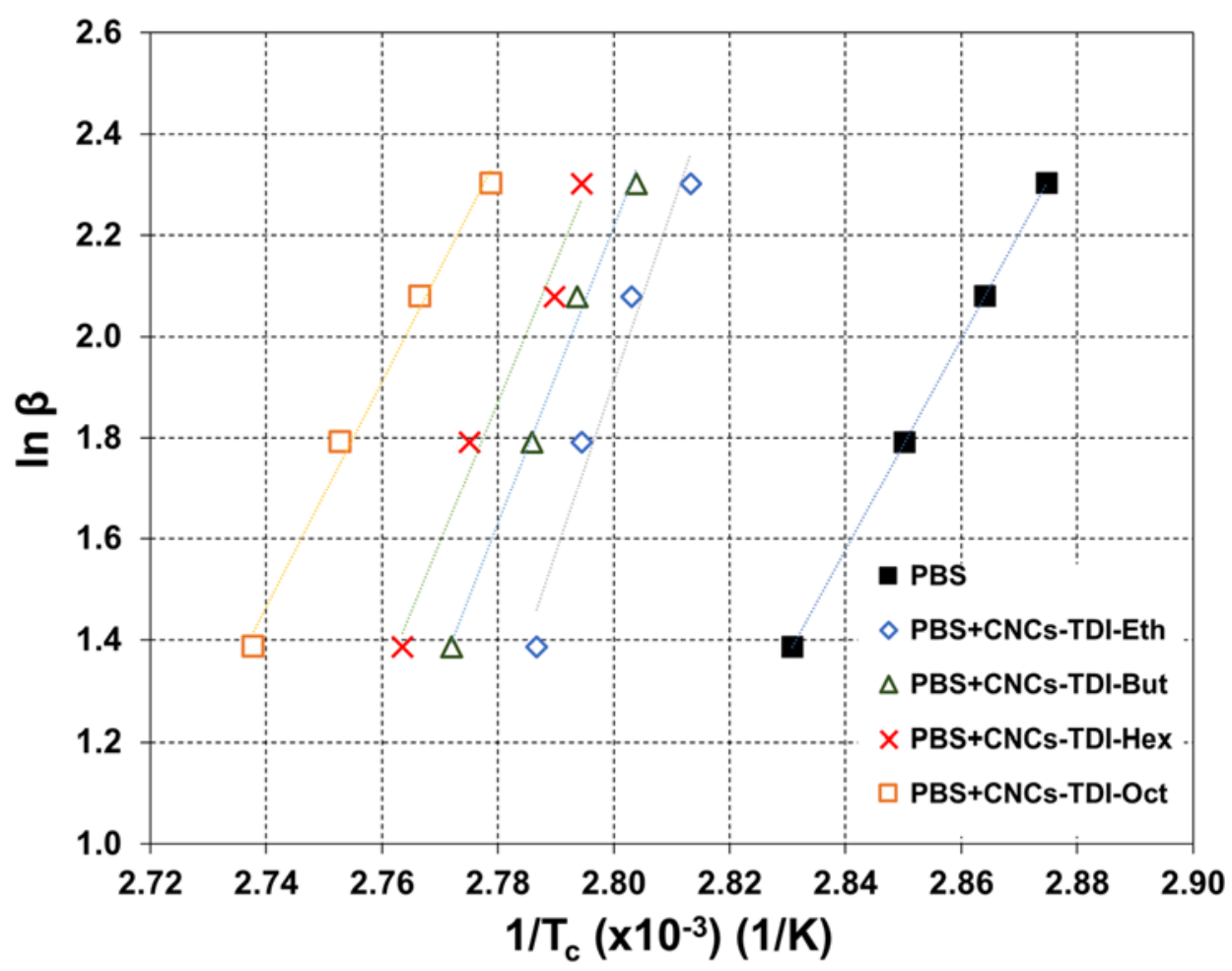

Figure 6. Kissinger plot to determine the activation energy of crystallization of neat and reinforced PBS.

In summary, the crystallization behavior of PBS was significantly affected by the addition of CNCs, which was dependent on their surface properties (Table 4). In general, CNC addition increased PBS crystallization temperature as a result of the nucleation activity of CNCs. The nucleation activity of the CNCs increased with the increase in their contact angle upon hydrophobization, which could be a result of the improved interaction between PBS and CNCs. Despite the significant improvement in PBS nucleation, its crystallization kinetics were slower as indicated by the drop of $Z$ value and increase of $t_{\frac{1}{2}}$ and $E_{a}$. This outcome indicates that the impeding effect of the CNCs was stronger than their nucleation activity, which as a result hindered the molecular motion of PBS chains and decelerated overall crystallization. However, the impeding effect of CNCs became less significant with reducing their hydrophilicity. In terms of the PBS melting temperature and crystallinity, the CNCs did not have a significant impact on them. 
Table 4. Summary of the impact of CNC surface properties on the crystallization of PBS (at $10^{\circ} \mathrm{C} / \mathrm{min}$ ).

\begin{tabular}{cccccccccc}
\hline Sample & Contact Angle $\left({ }^{\circ}\right)$ & $\mathbf{T}_{\mathbf{c}}\left({ }^{\circ} \mathbf{C}\right)$ & $\mathbf{T}_{\mathbf{m}}\left({ }^{\circ} \mathbf{C}\right)$ & $\mathbf{X}(\%)$ & $\mathbf{n}$ & $\mathbf{Z}$ & $\mathbf{t}_{\frac{1}{2}}(\mathbf{m i n})$ & $\mathbf{E}_{\mathbf{a}}(\mathbf{k J} / \mathbf{m o l})$ & $\boldsymbol{\phi}$ \\
\hline PBS & 77 & 74.7 & 115.7 & 56.5 & 3.8 & 0.39 & 1.1 & 169 & - \\
PBS+CNCs-TDI-Eth & $34 *$ & 82.2 & 115.8 & 53.4 & 3.3 & 0.29 & 1.4 & 266 & 0.82 \\
PBS+CNCs-TDI-But & $52 *$ & 83.4 & 115.9 & 53.3 & 3.5 & 0.32 & 1.3 & 236 & 0.63 \\
PBS+CNCs-TDI-Hex & $104 *$ & 84.5 & 115.8 & 59.2 & 3.0 & 0.33 & 1.1 & 225 & 0.52 \\
PBS+CNCs-TDI-Oct & $120 *$ & 86.6 & 115.9 & 61.0 & 3.4 & 0.40 & 1.1 & 196 & 0.35 \\
\hline \multicolumn{8}{c}{ * Contact angle of the CNCs not the composite. }
\end{tabular}

\section{Conclusions}

The impact of CNC surface properties on the crystallization behavior of PBS in CNC-reinforced PBS composites was investigated using differential scanning calorimetry. The surface properties of the CNCs were tailored by grafting alcohols of different chain lengths on their surface using TDI as a linker. The water contact angle of the modified CNCs increased to $34^{\circ}, 52^{\circ}, 104^{\circ}$, or $120^{\circ}$ using ethanol, 1-butanol, 1-hexanol, or 1-octanol, respectively. Compared to neat PBS, the crystallization thermograms of the PBS composites showed a heterogeneous crystallization behavior with increased crystallization temperature as a result of CNC nucleation activity, which was proportionally dependent on the contact angle of the CNCs. However, the estimated Avrami kinetic parameters and Kissinger activation energy of crystallization showed that the impeding effect of CNCs is prevailing, resulting in retarded crystallization. The impeding effect was less significant for the CNCs with higher contact angles. Overall, the surface properties of CNCs had a crucial role in the crystallization behavior of PBS and can be advantageous to tailor its properties.

Author Contributions: Conceptualization, H.A. and J.M.; methodology, H.A. and J.M.; formal analysis, H.A. and J.M.; investigation, H.A. and J.M.; writing-original draft preparation, H.A. and J.M.; writing-review and editing, H.A. and J.M.; funding acquisition, H.A. All authors have read and agreed to the published version of the manuscript.

Funding: This research was funded by the Fraunhofer Institute for Wood Research (WKI) through the Wilhelm-Klauditz Fellowship.

Acknowledgments: Many thanks to Nadine Nöcker from the Fraunhofer Institute for Surface Engineering and Thin Films (IST) for the contact angle measurements and to Martin Eichler from the Fraunhofer Institute for Wood Research (WKI) for performing the DSC measurements.

Conflicts of Interest: The authors declare no conflict of interest.

\section{References}

1. ISO. Standard Terms and Their Definition for Cellulose Nanomaterial; International Organization for Standardization (ISO): Geneva, Swizerland, 2017.

2. Abushammala, H.; Mao, J. A review on the partial and complete dissolution and fractionation of wood and lignocelluloses using imidazolium ionic liquids. Polymers 2020, 12, 195. [CrossRef]

3. Mao, J.; Abushammala, H.; Brown, N.; Laborie, M.P. Comparative assessment of methods for producing cellulose I nanocrystals from cellulosic sources. In Nanocelluloses: Their Preparation, Properties, and Applications, ACS Symposium Series; ACS Publications: Washington, DC, USA, 2017; Volume 1251, pp. 19-53.

4. Mao, J.; Abushammala, H.; Hettegger, H.; Rosenau, T.; Laborie, M.P. Imidazole, a new tunable reagent for producing nanocellulose, part I: Xylan-Coated CNCs and CNFs. Polymers 2017, 9, 473. [CrossRef] [PubMed]

5. Moon, R.J.; Martini, A.; Nairn, J.; Simonsen, J.; Youngblood, J. Cellulose nanomaterials review: Structure, properties and nanocomposites. Chem. Soc. Rev. 2011, 40, 3941-3994. [CrossRef] [PubMed]

6. Eichhorn, S.J.; Dufresne, A.; Aranguren, M.; Marcovich, N.E.; Capadona, J.R.; Rowan, S.J.; Weder, C.; Thielemans, W.; Roman, M.; Renneckar, S.; et al. Review: Current international research into cellulose nanofibres and nanocomposites. J. Mater. Sci. 2010, 45, 1-33. [CrossRef] 
7. Abushammala, H.; Mao, J. A review of the surface modification of cellulose and nanocellulose using aliphatic and aromatic mono- and di-isocyanates. Molecules 2019, 24, 2782. [CrossRef] [PubMed]

8. Eyley, S.; Thielemans, W. Surface modification of cellulose nanocrystals. Nanoscale 2014, 6, 7764-7779. [CrossRef] [PubMed]

9. Pirani, S.; Abushammala, H.M.; Hashaikeh, R. Preparation and characterization of electrospun PLA/nanocrystalline cellulose-based composites. J. Appl. Polym. Sci. 2013, 130, 3345-3354. [CrossRef]

10. Sapkota, J.; Natterodt, J.C.; Shirole, A.; Foster, E.J.; Weder, C. Fabrication and properties of polyethylene/cellulose nanocrystal composites. Macromol. Mater. Eng. 2017, 302, 1600300. [CrossRef]

11. Kargarzadeh, H.; Mariano, M.; Huang, J.; Lin, N.; Ahmad, I.; Dufresne, A.; Thomas, S. Recent developments on nanocellulose reinforced polymer nanocomposites: A review. Polymer 2017, 132, 368-393. [CrossRef]

12. Ferreira, F.V.; Dufresne, A.; Pinheiro, I.F.; Souza, D.H.S.; Gouveia, R.F.; Mei, L.H.I.; Lona, L.M.F. How do cellulose nanocrystals affect the overall properties of biodegradable polymer nanocomposites: A comprehensive review. Eur. Polym. J. 2018, 108, 274-285. [CrossRef]

13. Sinko, R.; Qin, X.; Keten, S. Interfacial mechanics of cellulose nanocrystals. MRS Bull. 2015, 40, 340-348. [CrossRef]

14. Inai, N.H.; Lewandowska, A.E.; Ghita, O.R.; Eichhorn, S.J. Interfaces in polyethylene oxide modified cellulose nanocrystal-polyethylene matrix composites. Compos. Sci. Technol. 2018, 154, 128-135. [CrossRef]

15. Lewandowska, A.; Eichhorn, S. Raman imaging as a tool for assessing the degree of mixing and the interface between polyethylene and cellulose nanocrystals. In Proceedings of the IOP Conference Series: Materials Science and Engineering 37th Risø International Symposium on Materials Science, Risø, Denmark, 5-8 September 2016.

16. Hu, F.; Lin, N.; Chang, P.R.; Huang, J. Reinforcement and nucleation of acetylated cellulose nanocrystals in foamed polyester composites. Carbohydr. Polym. 2015, 129, 208-215. [CrossRef] [PubMed]

17. Zhang, C.; Salick, M.R.; Cordie, T.M.; Ellingham, T.; Dan, Y.; Turng, L.S. Incorporation of poly(ethylene glycol) grafted cellulose nanocrystals in poly(lactic acid) electrospun nanocomposite fibers as potential scaffolds for bone tissue engineering. Mater. Sci. Eng. C 2015, 49, 463-471. [CrossRef] [PubMed]

18. Yu, H.Y.; Qin, Z.Y.; Yan, C.F.; Yao, J.M. Green nanocomposites based on functionalized cellulose nanocrystals: A study on the relationship between interfacial interaction and property enhancement. ACS Sustain. Chem. Eng. 2014, 2, 875-886. [CrossRef]

19. Abushammala, H. Nano-brushes of alcohols grafted onto cellulose nanocrystals for reinforcing poly(butylene succinate): Impact of alcohol chain length on interfacial adhesion. Polymers 2020, 12, 95. [CrossRef]

20. Ferreira, F.V.; Pinheiro, I.F.; de Souza, S.F.; Mei, L.H.; Lona, L.M. Polymer composites reinforced with natural fibers and nanocellulose in the automotive industry: A short review. J. Compos. Sci. 2019, 3, 51. [CrossRef]

21. Shi, J.; Xu, W.; Li, D.; Liao, R.; Zhang, L. The Innovation Research of Biodegradable Polymers for Sustainable Packaging. DEStech Trans. Environ. Energy Earth Sci. 2016. [CrossRef]

22. Gigli, M.; Fabbri, M.; Lotti, N.; Gamberini, R.; Rimini, B.; Munari, A. Poly (butylene succinate)-based polyesters for biomedical applications: A review. Eur. Polym. J. 2016, 75, 431-460. [CrossRef]

23. Xu, J.; Guo, B.H. Poly (butylene succinate) and its copolymers: Research, development and industrialization. Biotechnol. J. 2010, 5, 1149-1163. [CrossRef]

24. Bin, T.; Qu, J.P.; Liu, L.M.; Feng, Y.H.; Hu, S.X.; Yin, X.C. Non-isothermal crystallization kinetics and dynamic mechanical thermal properties of poly (butylene succinate) composites reinforced with cotton stalk bast fibers. Thermochim. Acta 2011, 525, 141-149. [CrossRef]

25. Zhang, G.; Xie, W.; Wu, D. Selective localization of starch nanocrystals in the biodegradable nanocomposites probed by crystallization temperatures. Carbohydr. Polym. 2020, 227, 115341. [CrossRef] [PubMed]

26. Pramoda, K.P.; Linh, N.T.T.; Zhang, C.; Liu, T. Multiwalled carbon nanotube nucleated crystallization behavior of biodegradable poly(butylene succinate) nanocomposites. J. Appl. Polym. Sci. 2009, 111, 2938-2945. [CrossRef]

27. Bosq, N.; Aht-Ong, D. Nonisothermal crystallization behavior of poly(butylene succinate)/NaY zeolite nanocomposites. Macromol. Res. 2018, 26, 13-21. [CrossRef] 
28. Li, Y.D.; Fu, Q.Q.; Wang, M.; Zeng, J.B. Morphology, crystallization and rheological behavior in poly(butylene succinate)/cellulose nanocrystal nanocomposites fabricated by solution coagulation. Carbohydr. Polym. 2017, 164, 75-82. [CrossRef] [PubMed]

29. Bao, L.; Chen, Y.; Zhou, W.; Wu, Y.; Huang, Y. Bamboo fibers@ poly (ethylene glycol)-reinforced poly (butylene succinate) biocomposites. J. Appl. Polym. Sci. 2011, 122, 2456-2466. [CrossRef]

30. Xu, J.; Manepalli, P.H.; Zhu, L.; Narayan-Sarathy, S.; Alavi, S. Morphological, barrier and mechanical properties of films from poly (butylene succinate) reinforced with nanocrystalline cellulose and chitin whiskers using melt extrusion. J. Polym. Res. 2019, 26, 188. [CrossRef]

31. Joy, J.; Jose, C.; Yu, X.; Mathew, L.; Thomas, S.; Pilla, S. The influence of nanocellulosic fiber, extracted from Helicteres isora, on thermal, wetting and viscoelastic properties of poly(butylene succinate) composites. Cellulose 2017, 24, 4313-4323. [CrossRef]

32. Phua, Y.; Pegoretti, A.; Araujo, T.M.; Ishak, Z.M. Mechanical and thermal properties of poly (butylene succinate)/poly (3-hydroxybutyrate-co-3-hydroxyvalerate) biodegradable blends. J. Appl. Polym. Sci. 2015, 132, 47. [CrossRef]

33. Chen, C.H. Effect of attapulgite on the crystallization behavior and mechanical properties of poly (butylene succinate) nanocomposites. J. Phys. Chem. Solids 2008, 69, 1411-1414. [CrossRef]

34. Liang, Z.; Pan, P.; Zhu, B.; Dong, T.; Inoue, Y. Mechanical and thermal properties of poly (butylene succinate)/plant fiber biodegradable composite. J. Appl. Polym. Sci. 2010, 115, 3559-3567. [CrossRef]

35. Han, H.; Wang, X.; Wu, D. Mechanical properties, morphology and crystallization kinetic studies of bio-based thermoplastic composites of poly (butylene succinate) with recycled carbon fiber. J. Chem. Technol. Biotechnol. 2013, 88, 1200-1211. [CrossRef]

36. Chen, G.X.; Yoon, J.S. Nonisothermal crystallization kinetics of poly (butylene succinate) composites with a twice functionalized organoclay. J. Polym. Sci. Part B Polym. Phys. 2005, 43, 817-826. [CrossRef]

37. Yarici, T.; Kodal, M.; Ozkoc, G. Non-isothermal crystallization kinetics of Poly (Butylene succinate)(PBS) nanocomposites with different modified carbon nanotubes. Polymer 2018, 146, 361-377. [CrossRef]

38. Bosq, N.; Aht-Ong, D. Isothermal and non-isothermal crystallization kinetics of poly (butylene succinate) with nanoprecipitated calcium carbonate as nucleating agent. J. Therm. Anal. Calorim. 2018, 132, 233-249. [CrossRef]

39. Filizgok, S.; Kodal, M.; Ozkoc, G. Non-isothermal crystallization kinetics and dynamic mechanical properties of poly (Butylene succinate) nanocomposites with different type of carbonaceous nanoparticles. Polym. Compos. 2018, 39, 2705-2721. [CrossRef]

40. Habibi, Y.; Dufresne, A. Highly filled bionanocomposites from functionalized polysaccharide nanocrystals. Biomacromolecules 2008, 9, 1974-1980. [CrossRef]

41. Abushammala, H. On the para/ortho reactivity of isocyanate groups during the carbamation of cellulose nanocrystals using 2,4-toluene diisocyanate. Polymers 2019, 11, 1164. [CrossRef]

42. Abushammala, H. A simple method for the quantification of free isocyanates on the surface of cellulose nanocrystals upon carbamation using toluene diisocyanate. Surfaces 2019, 2, 444-454. [CrossRef]

43. Correlo, V.M.; Boesel, L.F.; Bhattacharya, M.; Mano, J.F.; Neves, N.M.; Reis, R.L. Properties of melt processed chitosan and aliphatic polyester blends. Mater. Sci. Eng. A 2005, 403, 57-68. [CrossRef]

44. Dobreva, A.; Gutzow, I. Kinetics of Non-isothermal Overall Crystallization in Polymer Melts. Cryst. Res. Technol. 1991, 26, 863-874. [CrossRef]

45. Avrami, M. Kinetics of phase change. I General theory. J. Chem. Phys. 1939, 7, 1103-1112. [CrossRef]

46. Liu, Y.; Wang, L.; He, Y.; Fan, Z.; Li, S. Non-isothermal crystallization kinetics of poly (L-lactide). Polym. Int. 2010, 59, 1616-1621. [CrossRef]

47. Zhou, W.Y.; Duan, B.; Wang, M.; Cheung, W.L. Isothermal and non-isothermal crystallization kinetics of poly (L-Lactide)/carbonated hydroxyapatite nanocomposite microspheres. Adv. Divers. Ind. Appl. Nanocompos. 2011, 1, 231-260.

48. Ravari, F.; Mashak, A.; Nekoomanesh, M.; Mobedi, H. Non-isothermal cold crystallization behavior and kinetics of poly (1-lactide): Effect of l-lactide dimer. Polym. Bull. 2013, 70, 2569-2586. [CrossRef]

49. Papageorgiou, G.Z.; Achilias, D.S.; Bikiaris, D.N. Crystallization kinetics of biodegradable poly (butylene succinate) under isothermal and non-isothermal conditions. Macromol. Chem. Phys. 2007, 208, 1250-1264. [CrossRef] 
50. Seven, K.M.; Cogen, J.M.; Gilchrist, J.F. Nucleating agents for high-density polyethylene-A review. Polym. Eng. Sci. 2016, 56, 541-554. [CrossRef]

51. Calabia, B.P.; Ninomiya, F.; Yagi, H.; Oishi, A.; Taguchi, K.; Kunioka, M.; Funabashi, M. Biodegradable poly (butylene succinate) composites reinforced by cotton fiber with silane coupling agent. Polymers 2013, 5, 128-141. [CrossRef] 